livraisons

d'Histoire

de l'Architecture

\section{Livraisons de l'histoire de l'architecture}

41 | 2021

L'architecte en son agence

\title{
Les André, une stratégie professionnelle dynastique
}

Les André, a dynastic professional strategy

Die Familie André: eine dynastische Berufsstrategie

\section{Caroline Bauer}

\section{CpenEdition}

Journals

Édition électronique

URL : https://journals.openedition.org//ha/3240

DOI : 10.4000/lha.3240

ISSN : 1960-5994

Éditeur

Association Livraisons d'histoire de l'architecture - LHA

Référence électronique

Caroline Bauer, «Les André, une stratégie professionnelle dynastique », Livraisons de l'histoire de l'architecture [En ligne], 41 | 2021, mis en ligne le 15 juin 2021, consulté le 19 juin 2021. URL : http:// journals.openedition.org//ha/3240 ; DOI : https://doi.org/10.4000/lha.3240

Ce document a été généré automatiquement le 19 juin 2021.

Tous droits réservés à l'Association LHA 


\title{
Les André, une stratégie professionnelle dynastique
}

\author{
Les André, a dynastic professional strategy \\ Die Familie André: eine dynastische Berufsstrategie
}

\section{Caroline Bauer}

1 La famille André est une dynastie nancéienne dont on peut attester des liens avec le domaine de la construction depuis le XVIII ${ }^{\mathrm{e}}$ siècle. Né dans la seconde moitié du XVIII ${ }^{\mathrm{e}}$ siècle, Nicolas André (inc.) est appareilleur. Il a deux fils : François-Eugène (1816-1875), le cadet, est employé du ministère des Travaux publics, tandis que François (1811-1904), l'aîné, se forme dans les cours publics de sciences appliquées aux arts et métiers, et complète sa formation auprès de l'architecte Léon Adolphe Grillot. François André devient maître d'œuvre en 1830 pour des clients dont il est « le plus souvent à la fois l'architecte et l'entrepreneur ${ }^{1}$ ». Il épouse une fille d'un entrepreneur en bâtiment et devient père de quatre enfants. Son agence étend son emprise sur le territoire avec l'action du cadet, Charles André (1841-1928), qui reprend l'affaire familiale aux débuts de la III ${ }^{\mathrm{e}}$ République. Celle-ci sera active tout au long du XX $\mathrm{X}^{\mathrm{e}}$ siècle, avec l'implication des différentes générations: son fils Émile André (1871-1933), puis les deux frères Jacques (1904-1985) et Michel (1905-1975), puis Jean-Luc (né en 1939), et enfin Éric (né en 1963) actuellement toujours en activité (Ill. 1). 


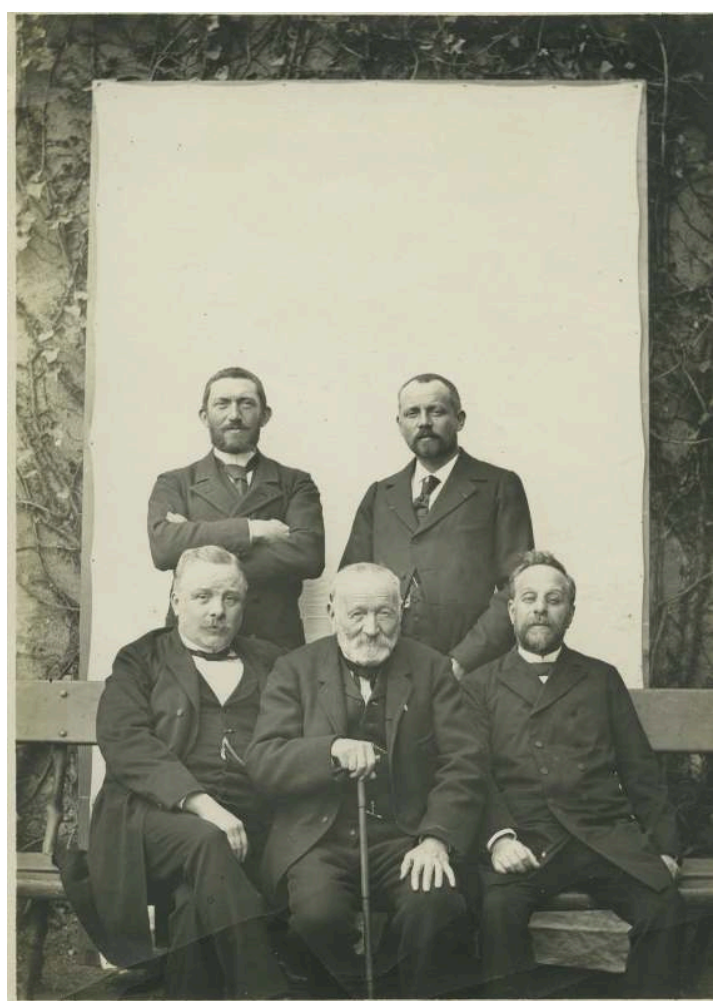

Entouré de ses quatre fils dont Charles André, architecte, assis à droite, s. d. (vers 1880).

Photographe anonyme, collection André.

2 Cette dynastie implantée dans la région lorraine met en place différentes stratégies pour pérenniser son activité et devenir l'une des plus importantes agences de la région au cours du $\mathrm{XX}^{\mathrm{e}}$ siècle. Dans un premier temps, nous verrons comment les André se sont appuyés sur le développement industriel de la ville de Nancy dans la seconde

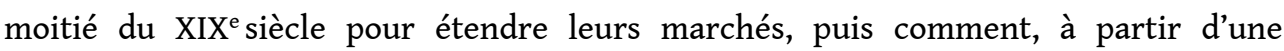
proximité avec la sphère politique municipale, ils ont opéré un retournement vers la commande étatique après la Seconde Guerre mondiale. Enfin, nous examinerons les outils de promotion qu'ils ont mis en place, du lieu même de l'agence à la diffusion des représentations de leur production ${ }^{2}$.

\section{Conquérir le nouveau marché de l'industrie}

3 À la suite de l'annexion de l'Alsace-Lorraine, la ville de Nancy devient la capitale de l'Est de la France et connaît, de 1870 à 1914, un important développement démographique et économique. Les industries locales, historiquement artisanales, évoluent en d'importants complexes industriels dans des domaines tels que la sidérurgie, la brasserie, la meunerie ou la faïencerie. Dès les années 1880, un mouvement collectif s'engage dans les arts décoratifs et se matérialise par la création de l'Alliance provinciale des industries d'art en 1901. Constituée autant d'artistes décorateurs et d'architectes, que d'industriels et d'intellectuels, cette association mieux connue sous le nom d'École de Nancy s'inscrit dans le mouvement de l'Art nouveau. Elle place ainsi la ville de Nancy comme un important foyer artistique au niveau européen, auquel Charles et Émile André participent. Charles en est l'un des plus grands 
promoteurs et s'y investit pour les générations suivantes. La renommée de la production de son fils, Émile André, s'appuie en effet sur sa participation à ce mouvement tout au long des années 1900. Émile André conjugue ses compétences d'artiste et de constructeur dans des réalisations aussi variées que les immeubles Huot (1903), la pharmacie Rosfelder (1905-1908) ou encore l'impressionnante banque Renauld (1905-1908), qui font aujourd'hui partie intégrante du patrimoine de la ville.

Dès les dernières décennies du XIX ${ }^{e}$ siècle, Charles et Émile André intègrent plus largement les réseaux des industriels; Charles André est en lien avec les membres de la Chambre de commerce qui regroupe de nombreux patrons et il est sollicité par les soudières Solvay. Mais c'est son fils Émile qui collabore régulièrement avec ce secteur à partir de 1905, date à laquelle il intègre la Société industrielle de l'Est. Probablement encouragé par le vote de la loi Paul Strauss en 1906, Émile André réalise ainsi plusieurs cités ouvrières pour Solvay, pour la société de construction automobile et ferroviaire De Dietrich, pour les soudières de la Madeleine ou encore pour la Société des carrières de Maxéville. Émile André s'éloigne à plusieurs reprises du dessin répétitif des cités ouvrières pour s'inspirer du modèle de cité-jardin proposé en 1898 par l'anglais Ebenezer Howard. Avec l'architecte Gaston Munier (1871-1918), il réalise ainsi un lotissement pour la société des eaux minérales de Vittel, et deux cités pour la Blanchisserie et teinturerie de Thaon dans les Vosges avec l'un des grands représentants de ce courant, l'architecte Jean Walter (1883-1957), qui voit également dans cette association la possibilité de développer son activité sur de nouveaux territoires.

5 Mais les interventions de Charles et Émile André pour les industriels portent essentiellement sur la construction de logements et d'équipements, et non sur les lieux même de production. En raison de la croissance importante du secteur, l'agence André met au point une stratégie spécifique pour accéder à ce nouveau marché. Le processus s'engage dans les années 1920 avec les deux fils d'Émile, Jacques et Michel André. En effet, alors que Jacques, l'aîné, se forme en tant qu'architecte à l'École des beaux-arts, son frère Michel, se dirige vers l'École centrale afin de devenir ingénieur. Michel André confie qu'il s'agit d'une alliance prévue en vue d'une collaboration ultérieure avec son frère Jacques André 3 . Alors qu'à l'École des beaux-arts, l'apprentissage de la composition domine, Michel acquiert des connaissances spécifiques dans le domaine de la technique et des matériaux en suivant par exemple des leçons sur la construction métallique ou le béton armé, particulièrement utiles dans les programmes très techniques des équipements industriels ${ }^{4}$.

6 Au-delà des compétences qu'il acquiert à l'École centrale, cette formation dont il sort diplômé en 1928, permet à Michel d'intégrer un cercle professionnel. En effet, les grands patrons d'industries, souvent centraliens, se reconnaissent dans son profil d'ingénieur. Michel intègre ainsi la branche locale du Rotary club, où se retrouvent ses condisciples diplômés de l'École centrale. L'association nancéienne, créée en 1929, est composée d'une majorité d'industriels, parmi lesquels les clients de l'agence, Antoine Trampitsch des Brasseries de Champigneulles, Marcel Vilgrain des Grands moulins du même nom, ou encore André Grandpierre de la société des Hauts-fourneaux et fonderies de Pont-à-Mousson. Cette association est placée sous la présidence d'Henri Brun, également président de la Société industrielle de l'Est.

7 Aucun contrat d'association ne lie les deux frères dans leur pratique professionnelle : Jacques André paye sa patente à partir de 1932, et Michel est, au niveau fiscal, 
l'employé de son frère ${ }^{5}$. Un partage des tâches se met en place naturellement et c'est Michel André qui s'occupe plus spécialement des questions techniques et des travaux industriels, même s'il n'exclut pas la direction de travaux particuliers (immeuble de rapport, villa...) et qu'il est par ailleurs chargé des programmes de logements sociaux. À la suite du décès de leur père Émile en 1933, les deux frères embauchent un ingénieur de la même génération qu'eux, Henri Haenel (1905-1998), qui collaborera une trentaine d'années avec eux. Dans la continuité du fonctionnement de l'agence qui semblait employer, au lendemain de la Première Guerre mondiale, deux dessinateurs, un métreur-vérificateur et une dactylographe, plusieurs autres collaborateurs devaient compléter ce noyau ${ }^{6}$.

Dès les années 1930, l'agence André réalise ainsi la construction, l'aménagement ou l'extension de plusieurs usines et leur activité s'intensifie après la Seconde Guerre mondiale. Intervenant dans des secteurs diversifiés (alimentaire, textile, cristalleries, presse...), les deux frères travaillent pour plusieurs compagnies sidérurgiques et deviennent ainsi les architectes attitrés de l'importante Compagnie des forges de Chatillon-Commentry et Neuves-Maisons, tout en intégrant plus largement les institutions qui leur sont liées. En 1937, ils deviennent ainsi les architectes de la Société nancéienne de Crédit industriel et de Dépôts (SNCI), qui se constitue dès ses débuts comme l'une des principales banques de la sidérurgie lorraine, puis, en 1943, ils deviennent ceux de la Chambre de commerce de Nancy. La reconnaissance de l'agence dans le secteur sidérurgique leur permettra d'être sollicités pour la création d'un projet muséal dédié à l'Histoire du fer et largement financé par des industriels locaux. Avec la réalisation de ce musée à Jarville, près de Nancy, ils remportent en 1969 le prix national de l'Équerre d'argent.

\section{Savoir s'adapter : du privé au public, de l'échelon municipal aux ministères}

9 L'agence André développe une part importante de son activité grâce à une clientèle privée, notamment de grandes industries, mais elle s'implique aussi dans la construction publique, en particulier aux côtés de la municipalité nancéienne. Dans la sphère locale, les André s'appuient sur un réseau professionnel qu'ils entretiennent de décennie en décennie, en particulier aux côtés de l'entreprise France-Lanord et Bichaton, et des artistes et constructeurs de la famille Prouvé 7 . À partir du milieu du XIXe siècle, la famille André s'investit surtout dans la vie politique nancéienne. Ses membres y sont ainsi présents durant près de 75 ans: François et Charles sont conseillers municipaux sur une période allant de 1848 à 1904 et Émile André reprend le flambeau de 1912 à 1925. À partir de 1925, les André conservent uniquement des appuis au sein du conseil municipal mais ne s'y impliquent plus directement.

10 Cette proximité avec le monde politique local semble avoir profité en premier lieu à Charles André qui, malgré son absence de formation Beaux-arts, est nommé architecte départemental en 1886. Il réalise à ce titre des casernes, un hospice, un tribunal, aménage la préfecture, les archives départementales et la maison de secours, et agrandit une école d'institutrices. Émile André, son fils, profite quant à lui de son mandat pour relancer les débats sur l'enseignement artistique, au moment où ses deux fils, Jacques et Michel, sont sur le point d'intégrer l'enseignement supérieur. L'un des effets les plus manifestes de cette proximité avec le réseau politique local concerne 
probablement les études préliminaires pour le transfert du musée de peinture et de sculpture de Nancy. Ce travail est en effet confié par la municipalité à Jacques André, alors qu'il n'est encore qu'un élève des Beaux-arts de 24 ans. Associés dès les prémisses du projet, Jacques et Michel accompagnés de leur père Émile remporteront ainsi le concours que la Ville lance en 1931.

11 Après la Seconde Guerre mondiale, l'agence se voit contrainte d'adapter sa stratégie de commande publique à une autre échelle. En effet, les importants besoins liés à la reconstruction sont pris en charge au niveau étatique. Dès les premiers mois du conflit, Jacques André est approché par un ancien commanditaire pour réaliser des projets d'infirmeries, d'hôpitaux et d'écoles pour les ministères de la Santé publique, de l'Éducation nationale et de l'Intérieur. Jacques André semble également avoir établi un rapprochement avec Raoul Dautry, qu'il ambitionne de rencontrer en 1939 lorsqu'il est ministre de l'armement ${ }^{8}$. À sa nomination à la tête du Ministère de la Reconstruction et de l'Urbanisme (MRU) en novembre 1944, Jacques André obtient d'ailleurs directement du ministère l'importante commande de la reconstruction de la ville de Saint-Dié, aux dépens d'un architecte proposé par le département.

Grâce à son ancrage lorrain, à son importance, mais aussi aux compétences techniques de Michel André, l'agence multiplie ses interventions auprès des ministères. Afin de gérer l'organisation des services de la construction, les administrations mettent en place, à partir des années 1940, des agréments et des postes d'architectes attitrés. Jacques André va cumuler les postes, assurant à l'agence une alimentation continue en projets: il obtient l'agrément du ministère de l'Éducation nationale en 1943, il est désigné architecte des Bâtiments civils et Palais nationaux en 1948 et architecte des Postes télégraphes et téléphones pour la région lorraine en 1951.

L'agence augmente ainsi le nombre de ses collaborateurs et agrandit ses locaux de la Place Stanislas, en aménageant les greniers. De manière assez stable, elle est constituée durant toute la seconde moitié du XX $\mathrm{XX}^{\mathrm{e}}$ siècle de douze à quinze collaborateurs, et se place ainsi comme l'une des plus importantes agences nancéiennes. Henri Haenel, le fidèle collaborateur qui a été recruté dans les années 1930, poursuit son activité au sein de l'agence jusque dans les années $1960^{\circ}$. Un nouveau chef d'agence est également recruté après la guerre, Georges Recher (1907-?). Jacques et Michel André accordent une totale confiance à leurs deux collaborateurs : chacun est responsable d'affaires qui lui sont propres et en assure le chantier. Ils s'adjoignent les services d'un ensemble de salariés, regroupés au sein d'un bureau de dessin et d'un bureau de métré, ainsi que d'une secrétaire.

\section{Développer ses outils de promotion}

La pérennité de l'agence peut aussi s'expliquer par un maintien constant de sa notoriété. Pour asseoir sa réputation, la dynastie André est attentive aux outils de sa promotion. Au tournant de l'année 1901, elle opère en particulier un choix judicieux pour l'implantation de ses locaux. Alors que Charles André devait probablement exercer au sein de son habitation, Émile André installe en effet l'agence au numéro un de la place Stanislas, construite en l'honneur de Louis XV entre 1753 et 1755, et fleuron patrimonial de la ville. Alors que les autres pavillons sont consacrés à des fonctions liées à la vie politique, culturelle et administrative de la cité, Émile André occupe le pavillon Jacquet, le seul qui est originellement destiné à un particulier. Installés dans 
l'attique, dans l'angle sud-ouest de la place, les locaux de l'agence disposent d'un emplacement privilégié au cœur de la ville. L'agence jouit par ailleurs de cette adresse prestigieuse qui pare la plupart de leurs tampons et papiers à en-tête. Les bureaux mêmes de l'agence font l'objet d'une attention particulière. Au début des années 1900, Emile André dessine une impressionnante table à dessin zoomorphe ainsi qu'un plafonnier en fer forgé, qui resteront en place jusqu'au siècle suivant. Au milieu des années 1930, après la reprise exclusive de l'agence par les deux frères, Jacques André conçoit un nouveau mobilier de travail, pour lequel il emploie le bois dans des compositions simples mais justement proportionnées. C'est dans ce nouvel agencement que les deux frères se mettront en scène au sein d'un reportage photographique (Ill. 2).

\section{2 : Jacques André et Michel André}

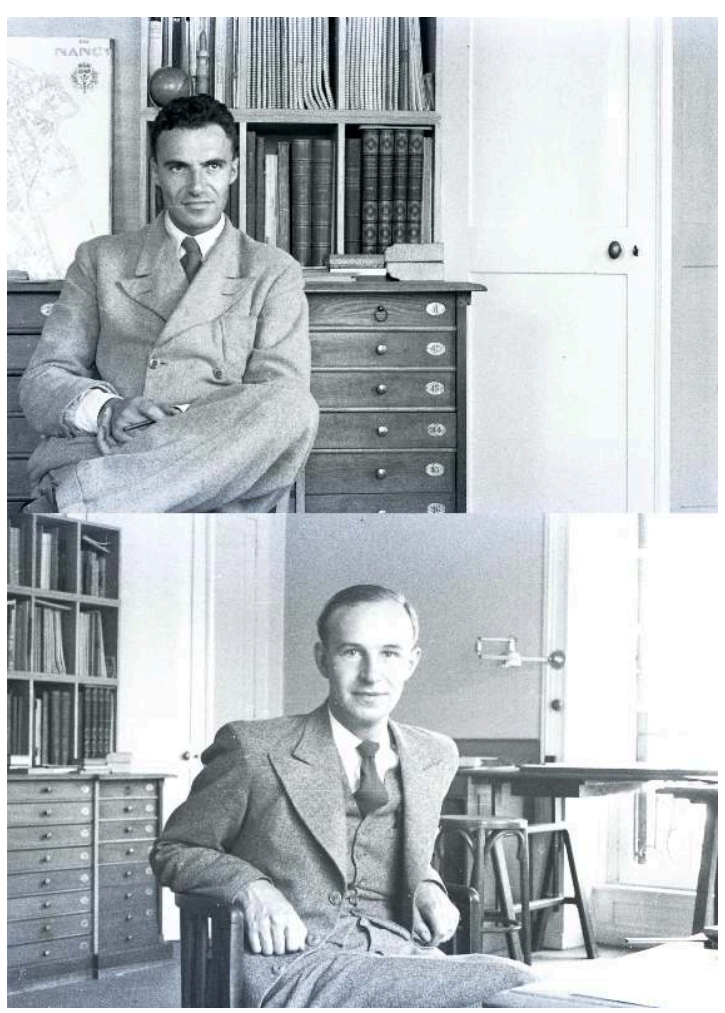

Jacques André (en haut) et Michel André (en bas), dans leur agence au 1, place Stanislas à Nancy, s. d. (vers 1935).

Photographe anonyme, collection André

Quelques années après l'installation de ses bureaux place Stanislas, Émile André décide d'aménager son logement personnel dans les espaces contigus à l'agence. A la mort de son père en 1933, Jacques André s'y installe à son tour, avec son épouse et ses trois enfants. Il fait de son appartement personnel un lieu de convivialité, où le cercle local se retrouve, mais aussi un lieu de promotion de la modernité. Son aménagement fait d'ailleurs l'objet d'un article paru dans La Maison française; la revue met en avant le contraste réussi entre la modernité des installations et le cadre classique de la place Stanislas ${ }^{10}$. L'harmonie des couleurs règne dans cet appartement, dans un esprit fidèle aux réalisations des deux frères : les murs du living-room sont d'un revêtement de bois collé clair, la salle à manger d'un bleu pâle mat, et le vestibule est recouvert d'un papier jaune à pois blancs. À ces teintes, s'ajoutent les motifs colorés des tissus des rideaux, dessinés par les créateurs Suzanne Fontan et Bauret-Warin. Pour la décoration, les 
objets recueillis par Émile André au cours de ses voyages agrémentent les espaces : miniatures, bronzes et tapis persans. Si plusieurs pièces de mobilier ont aussi été dessinées par Jacques André, il possède également une chaise de type LC4 réalisée par Charlotte Perriand, Le Corbusier et Pierre Jeanneret (Ill. 3 et 4).

III. 3 et 4 : extrait de l'article consacré à l'aménagement de l'appartement de Jacques André

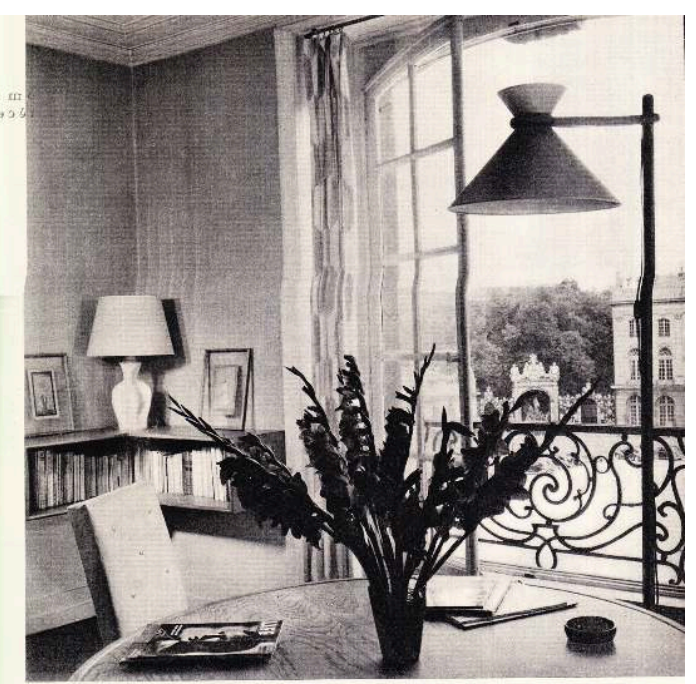

Comme tolle de fond: la place Stanislas..

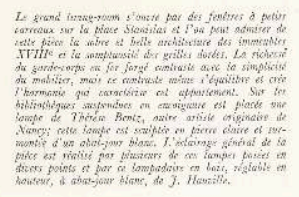




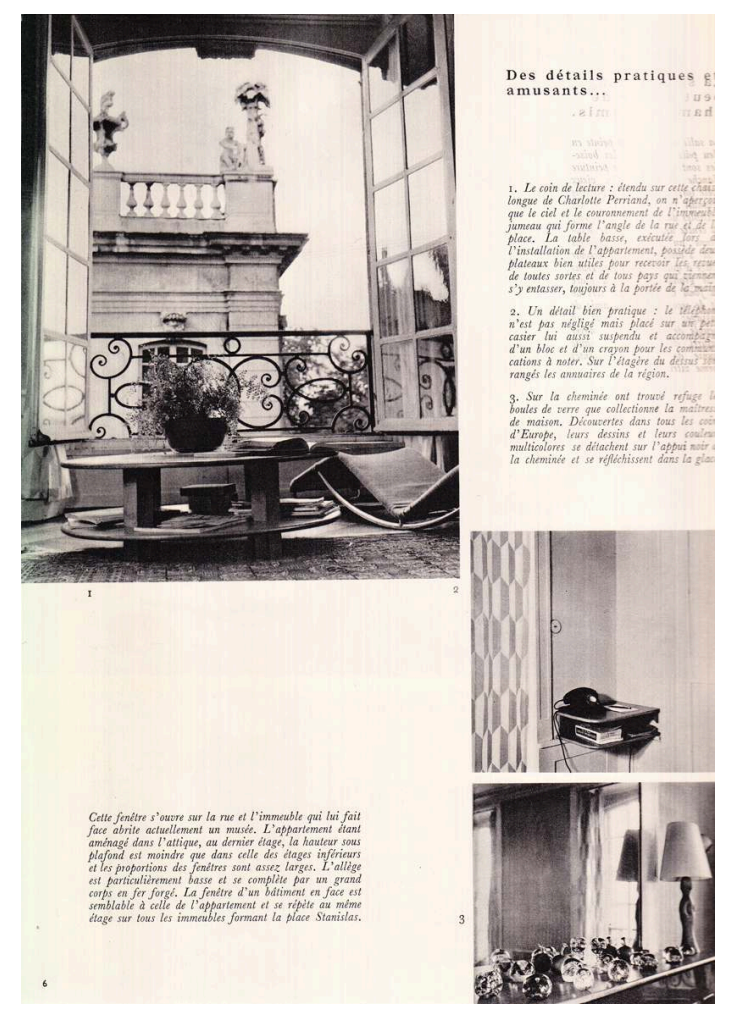

Extrait de l'article consacré à l'aménagement de l'appartement de Jacques André, place Stanislas à Nancy, «À Nancy, cadre classique, logis moderne. Jacques André, architecte », La Maison française, $n^{\circ} 71$, octobre 1953, p. 5-6.

D. R.

16 Au-delà de la maîtrise des lieux de représentation, la notoriété grandissante de Jacques et Michel André dans les années 1930 les entraîne à prendre en main la communication de leur travail. Dans le climat de raréfaction de la commande dû à la crise, il semble indispensable de posséder un outil efficace d'autopromotion. Après seulement quelques années d'exercice, Jacques et Michel André font appel aux éditions Batimod pour réaliser un ouvrage constitué de près d'une trentaine de planches de photographies ${ }^{11}$. A travers les quatre projets présentés, deux grandes commandes publiques et deux ensembles d'habitation, les frères André apparaissent comme des architectes capables de répondre tant à des particuliers qu'à des municipalités désireuses d'édifier des opérations d'envergure. Si l'auto publication n'est pas une pratique isolée, l'ouvrage des André se démarque sur le territoire nancéien. L'aspect extrêmement dépouillé, vaste et clair des salles de sculpture du musée de Nancy contraste avec les intérieurs très décorés des maisons cossues des autres albums d'architectes. Le souhait de s'inscrire dans le courant moderne est également perceptible dans les choix photographiques, comme l'exprime une vue en contre-plongée d'une maison individuelle mettant particulièrement en avant l'utilisation des pilotis.

Les images de certains édifices réalisés par les membres de la famille André deviennent elles-mêmes des outils de promotion. C'est le cas, en particulier, pour l'institut de zoologie réalisé en 1932-1933. Les frères André y réinterprètent un procédé de blocbéton de Frank Lloyd Wright directement lisible en façade. Celle-ci multiplie un motif de carré emboité sur un linéaire de plus de 70 mètres et arbore une couleur rosée dû à un mélange de pierre rose et de grains en marbre blanc ${ }^{12}$. Cette façade qui concentre l'essence du projet devient alors une de ses représentations emblématiques (Ill.5). 
L'édifice bénéficie alors rapidement de nombreux articles dans les revues françaises, qui soulignent les qualités de composition de la façade et en détaillent les aspects constructifs $^{13}$. Grâce à Jean Prouvé, collaborateur et ami des André, la revue L'Architecture d'aujourd'hui publie en 1934 un article de six pages sur l'édifice, illustré de plans et photographies ${ }^{14}$. Cette visibilité au sein d'une des revues les plus influentes de son époque provoque un intérêt au-delà des frontières. Dexter Morand, correspondant des États-Unis pour L'Architecture d'aujourd'hui, souhaite publier des articles dans la revue anglaise The Building Times et dans la revue américaine Architectural Forum ${ }^{15}$. Des membres de leur comité ayant vu des photographies du bâtiment, le Royal Institute of British Architects (RIBA) de Londres souhaite intégrer une illustration à ses collections permanentes ${ }^{16}$.

\section{5 : Institut de zoologie à Nancy}

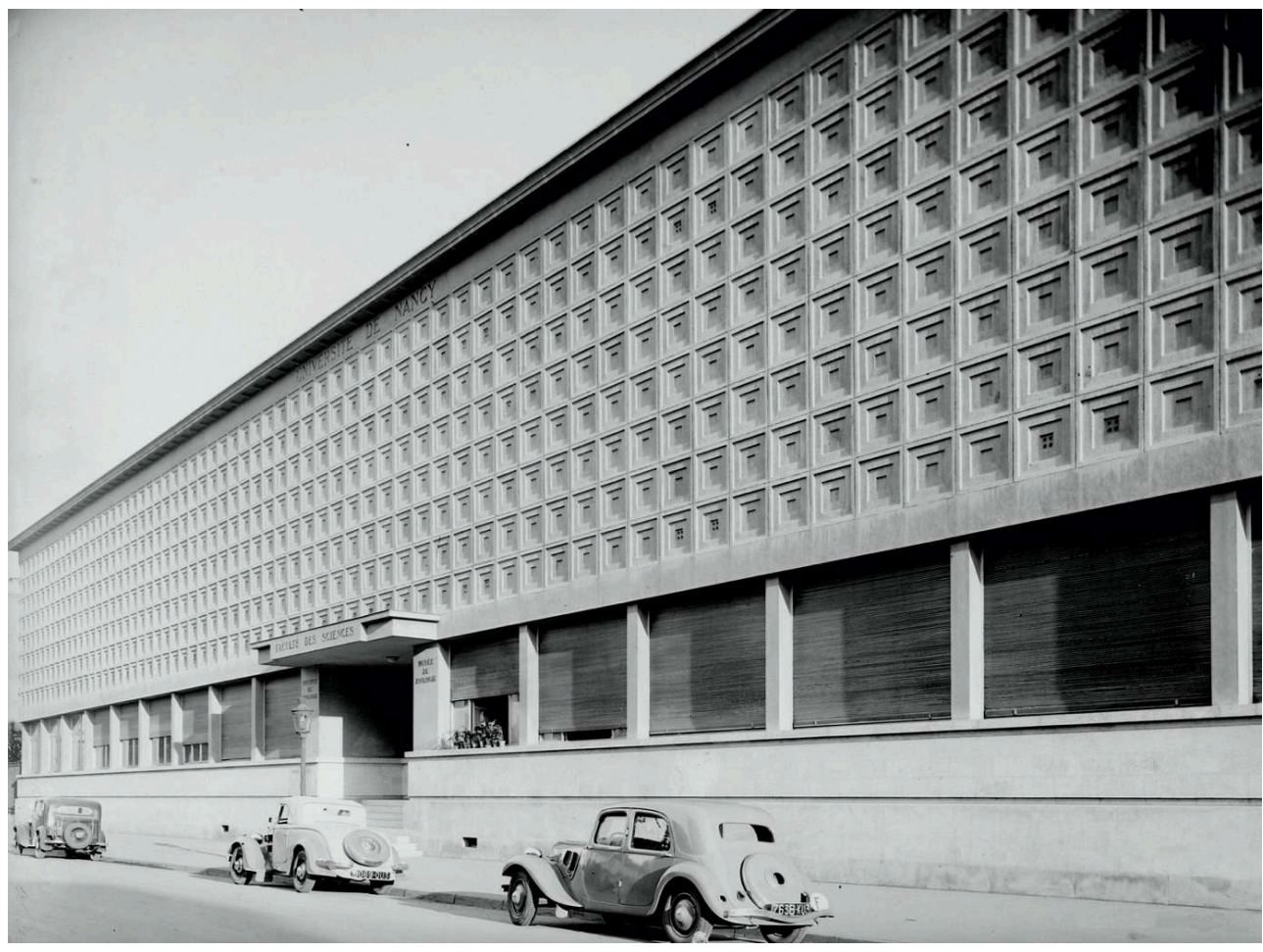

Jacques André et Michel André, Institut de zoologie à Nancy, s.d. (vers 1933).

Photographe anonyme, collection Image'Est.

18 L'Institut de zoologie est alors considéré comme un édifice de référence des années 1930 et représente l'architecture moderne française à l'international pendant plusieurs années. Il intègre le choix de photographies que réunit L'Architecture d'aujourd'hui pour les exposer au sein du pavillon international de l'Exposition de Bruxelles en 1935. Par le biais de Pierre Vago, rédacteur en chef de la revue, le bâtiment est encore une fois exposé à la Triennale de Milan en 1936, ou encore au pavillon de l'Union des Artistes Modernes de l'exposition internationale de 1937. Même après-guerre, il est présenté dans une exposition itinérante consacrée à l'architecture française organisée par la revue Techniques et architecture qui est inaugurée à Berne en 1946, et doit être reconduite en Tchécoslovaquie, Pologne, Hongrie, Autriche et dans les Pays scandinaves ${ }^{17}$. 
19 Après plusieurs décennies d'activité et sept générations de constructeurs, l'agence André est, en 2021, toujours en activité dans les mêmes locaux de la place Stanislas. Cette pérennité est le résultat des capacités d'adaptation de ses membres, tant aux différents contextes de production qu'aux fluctuations des besoins d'une clientèle. Sa réussite tient également à ses collaborations avec entrepreneurs et artistes, et encore plus à des associations décisives qui ont fait évoluer sa structure même. L'association de Jacques, Michel et Jean-Luc André avec Claude Prouvé en 1966, marque ainsi une étape importante, en scellant une relation ancienne entre les familles André et Prouvé, permettant de profiter de la notoriété supplémentaire associée au nom de Jean Prouvé. D'autres associations ont par la suite égrené son parcours, permettant à l'actuelle agence André-Moulet d'être le bureau d'architecture dont le chiffre d'affaires est le plus important de Nancy et, au-delà, du département de Meurthe-et-Moselle ${ }^{18}$.

\section{NOTES}

1. Émile Jacquemin, « Notre famille du bâtiment ", L'Immeuble et la construction dans l'Est, $n^{\circ} 33,11$ décembre 1904, p. 261.

2. Cet article est issu des recherches menées dans le cadre d'un travail de thèse de doctorat, dont l'objectif était d'étudier tant la production architecturale d'une agence que son fonctionnement, au prisme de ses réseaux et de ses filiations. Le fonds d'archives de l'agence conservé aux archives départementales de Meurthe-et-Moselle a constitué une importante source de documentation, mais principalement d'ordre graphique. Les dossiers personnels conservés dans les archives des administrations pour lesquelles l'agence avait travaillé, ainsi que ceux conservés par le Conseil régional de l'Ordre des architectes, ont permis d'éclairer davantage les processus de travail. Ils ont notamment permis de pallier à l'absence de consultation d'archives spécifiquement administratives de l'agence. Enfin, une campagne d'entretiens avec Jean-Luc André, associé à partir de 1966, et de plusieurs collaborateurs et fils de collaborateurs ont permis de compléter les connaissances sur son fonctionnement. C. Bauer, L'agence André au temps de Jacques et Michel (Nancy, 1929-1973). Architecture, réseaux et filiations, thèse de doctorat en histoire de l'art, sous la direction de Claude Massu, Paris 1 Panthéon-Sorbonne, 2015, 682 p., $339 \mathrm{pl}$.

3. Lettre de Michel André à Paul Charbonnier, président de Conseil régional de l'Ordre des architectes, 31 décembre 1941. Dossier de Michel André, Archives du Conseil régional de l'Ordre des architectes de Lorraine (CROAL).

4. Caroline Bauer, « ̀̀ la conquête du milieu sidérurgique : la stratégie de formation architecte/ ingénieur de Jacques André (1904-1985) et Michel André (1904-1975) », dans HEnsA20, Histoire de l'enseignement de l'architecture au $\mathrm{XX}^{e}$ siècle, Comité d'histoire et Service de l'architecture de la direction générale des patrimoines, ministère de la culture et de la communication, $\mathrm{n}^{\circ} 2$, novembre 2016, p. 31-36. Consultable en ligne : https://chmcc.hypotheses.org/3123

5. Ce statut entrânera des difficultés au moment de la création de l'Ordre des architectes le 30 décembre 1940. Si, en tant qu'architecte diplômé de l'École des beaux-arts de Paris, Jacques André n'a à effectuer qu'une simple demande pour s'inscrire, il faudra attendre le décret paru au Journal Officiel du 14 janvier 1942 pour que les diplômés de l'École centrale de 1924 à 1939, section construction, qui n'ont pas payé la patente d'entrepreneur, aient l'autorisation de s'inscrire à l'Ordre des architectes. 
6. Selon l'annonce parue dans L'Est Républicain, 31 décembre 1918.

7. Caroline Bauer, «Entre local et (inter)national, le rôle des réseaux dans la commande architecturale (Nancy, XIX ${ }^{\mathrm{e}} \mathrm{XX} \mathrm{X}^{\mathrm{e}}$ siècle) », dans Bienvenu Gilles, Monteil Martial et RousteauChambon Hélène, Construire! Entre Antiquité et Époque contemporaine, actes du troisième congrès francophone d'histoire de la construction, Nantes, 21-23 juin 2017, Paris, Picard, 2019, 1314 p., p. 843-853.

8. Ces réseaux ont pu être mis à jour en partie grâce à une correspondance entre les deux frères entre 1939 à 1941, lorsque Michel est prisonnier dans un camp autrichien. Archives famille André.

9. Il semble que ce soit Pierre Lequerme qui le remplace après son départ.

10. «À Nancy, cadre classique, logis moderne. Jacques André, architecte », La Maison française, n' 71, octobre 1953 , p. 3-7.

11. Jacques André et Michel André. Architecture, 1933-1936, Éditions d'art Batimod, Strasbourg, s. d. (1936), $29 \mathrm{p}$.

12. Caroline Bauer, «Le mur aveugle, transformer la contrainte en support d'innovation. L'Institut de zoologie, Jacques et Michel André, Nancy, 1932-1933» dans Marie Gaimard, Élise Guillerm, Claude Massu (dir.), Histo.art 5, Métier : architecte. Dynamiques et enjeux professionnels au cours du XXe siècle, Paris, Publications de la Sorbonne, 2013, 358 p., p. 53-67.

13. Par exemple, S. Duchêne, "Institut de zoologie de l'Université de Nancy et Musée de zoologie ", La construction moderne, $\mathrm{n}^{\circ}$ 7, 18 novembre 1934, p. 154. et A. B., " Musée et institut de zoologie à Nancy. J. et M. André, architectes ", L'Architecture d'Aujourd'hui, décembre 1934-janvier 1935 , p. 43.

14. André Bloc, «Musée et Institut de zoologie. J. et M. André, architectes ", L'Architecture d'aujourd'hui, $\mathrm{n}^{\circ}$ 10, décembre 1934-janvier 1935, p. 42-47.

15. Un article est paru dans la revue The Building Times, «Zoological Institute and Museum, Nancy, France. Architects : E. J. \& M. André », juin 1935, p. 8-9, ainsi qu'un autre dans la revue Architect \& building news, "Institute and Zoological Museum at Nancy, France », 2 août 1935, p. 144-146.

16. Elle sera publiée dans le Journal of the Royal Institute of British Architects, « Musee et Institut de Zoologie, Nancy; Architects: Michel \& Jacques Andre », 8 mai 1937, p. 670.

17. André Bouxin, «Exposition d'architecture française », Techniques et Architecture, n 5-6, 1946, p. 259.

18. Avec 1636000 euros de chiffre d'affaires en ;2015, elle se classe à la $334^{\mathrm{e}}$ position des agences françaises. Dans «Les 350 premières agences françaises classées selon leurs chiffres d'affaires ", D’A, n² 232, décembre 2014/janvier 2015, p. 176.

\section{RÉSUMÉS}

Présente sur le territoire lorrain depuis le XVIII ${ }^{\mathrm{e}}$ siècle et toujours en activité dans les mêmes locaux de la place Stanislas à Nancy, la dynastie de constructeurs et architectes André est un modèle de stratégie professionnelle. Chacune de ses générations s'adapte aux différents contextes de production comme aux fluctuations des besoins d'une clientèle. Dans la seconde moitié du XIX ${ }^{e}$ siècle, elle s'appuie ainsi sur le développement de l'industrie pour étendre ses marchés, grâce à l'acquisition de nouvelles compétences en ingénierie. Proches des cercles 
politiques, essentiellement dans la sphère municipale jusqu'aux années 1920, l'agence opère un retournement vers la commande étatique après la Seconde Guerre mondiale en multipliant les nominations et les agréments pour les ministères. L'agence apporte également un grand soin à sa représentation, que ce soit à travers le lieu même de l'agence et de l'appartement qui lui est contigu, qu'à la circulation des images de leurs réalisations.

Present in Lorraine since the 18th century and still operating in the same premises on Place Stanislas in Nancy, the André dynasty of builders and architects is a model of professional strategy. Each of its generations adapts to different production contexts and to the fluctuating needs of a clientele. In the second half of the 19th century, the dynasty relied on the development of the industry to expand its markets, thanks to the acquisition of new engineering skills. Close to political circles, essentially in the municipal sphere until the 1920s, the agency made a turnaround towards state control after the Second World War by multiplying appointments and approvals for government departments. The agency also takes great care in its representation, both through the location of the agency and the apartment adjacent to it, and in the circulation of images of their work.

Die seit dem 18. Jahrhundert in Lothringen nachzuweisende und immer noch in denselben Räumlichkeiten an der Place Stanislas in Nancy tätige Architektenfamilie André soll in diesem Beitrag als Beispiel einer Berufsstrategie vorgestellt werden. Jeder Generation gelang es auf eigene Weise, sich den jeweiligen Produktionsgegebenheiten und den jeweiligen Kundenwünschen anzupassen. In der zweiten Hälfte des 19. Jahrhunderts setzte die Familie im Zuge der ingenieurstechnischen Entwicklungen auf eine zunehmende Industrialisierung, um ihr Wirkungsfeld zu erweitern. Bis in die 1920er Jahre pflegte das Architekturbüro enge Beziehungen vor allem zur Lokalpolitik, weitete diese jedoch nach dem Zweiten Weltkrieg mehr und mehr auf staatliche Aufträge aus, wovon zahlreiche Arbeiten für verschiedene Ministerien zeugen. Das Prestige des Architekturbüros André spiegelt sich sowohl in der Ausstattung der zugehörigen Räumlichkeiten wider als auch in der medialen Verbreitung der durchgeführten Aufträge.

\section{INDEX}

Mots-clés : architecte, ingénieur, profession, stratégie professionnelle, agence, industrie, Nancy, Art nouveau, École de Nancy, André, Prouvé, politique, commande publique, commande privée

\section{AUTEUR}

\section{CAROLINE BAUER}

Titulaire du diplôme d'architecte DPLG (ENSA Nancy, 2007) et docteure en histoire de l'art (Université Paris 1 Panthéon-Sorbonne, 2015), Caroline Bauer est maître de conférences en Histoire et cultures architecturales à l'École nationale supérieure d'architecture et de paysage de Lille. Chercheuse au Laboratoire conception territoire histoire matérialité (LACTH) de l'ENSAP Lille, chercheuse associée au Laboratoire d'histoire de l'architecture contemporaine (LHAC) de l'ENSA Nancy, elle est également membre du conseil d'administration et du conseil scientifique de Docomomo France, association pour la documentation et la conservation des édifices et sites du Mouvement Moderne. Ses recherches portent sur l'architecture, le patrimoine et la profession d'architecte au XX siècle. Adresse électronique : c-bauer@lille.archi.fr 